\title{
A case report of SPG11 mutations in a Chinese ARHSP-TCC family
}

Linwei Zhang ${ }^{1,2}$, Karen N. McFarland ${ }^{2}$, Jinsong Jiao ${ }^{1}$ and Yujuan Jiao ${ }^{1 *}$

\begin{abstract}
Background: Autosomal recessive hereditary spastic paraplegia (ARHSP) with thin corpus callosum (TCC) is a complicated form of hereditary spastic paraplegia, characterized by progressive spastic paraplegia, weakness of the lower extremities and is usually accompanied by mental retardation. Mutations in the Spastic Paraplegia gene 11 (SPG11) account for a large proportion of ARHSP-TCC cases worldwide.

Case presentation: We describe a Chinese family with ARHSP-TCC. Two daughters of this family presented with a spastic gait and cognitive impairment. Brain imaging of the index patient revealed a thin corpus callosum. We performed detailed physical and auxiliary examinations and were able to exclude acquired causes of spastic paraplegia. To determine the causative mutation, we took a candidate gene approach and screened the coding sequence and some flanking intronic sequence of SPG11 by direct Sanger sequencing. We identified two novel compound heterozygous mutations in SPG11 in affected individuals (c.1551_1552delT, p.Cys518SerfsTer39 and c.5867-1G > T (IVS30-1G > T), p. Thr1956ArgfsTer15). Bioinformatic analysis predicts that these mutations would lead to a loss of protein function due to the truncation of the SPG11 protein.

Conclusions: The results of this case report indicate a broader approach to include screening for SPG11 mutations in ARHSP-TCC patients. Our findings enrich the phenotypic spectrum of SPG11 mutations.
\end{abstract}

Keywords: Autosomal recessive hereditary spastic paraplegia with thin corpus callosum (ARHSP-TCC), SPG11, Gene mutation, Heterozygous mutations

\section{Background}

Hereditary spastic paraplegia (HSP) is a kind of neurodegenerative disease characterized by progressive weakness and spasticity of the lower limbs. Depending on the mode of inheritance, it can be classified as autosomal dominant, autosomal recessive or X-linked. In complicated forms, additional neurological signs, such as ataxia, mental retardation, epilepsy, peripheral neuropathies, are present [1]. HSP with Thin Corpus Callosum (HSP-TCC) is one of the most common complicated forms of autosomal recessive hereditary spastic paraplegia (ARHSP). ARHSP-TCC is most commonly caused by mutations in the spastic paraplegia 11 (SPG11) gene [2]. SPG11 maps to chromosome 15q21, encodes a 2443 amino acid protein, SPATACSIN, and is widely expressed in the nervous system, particularly in the neurons of the cerebellum and cerebral cortex [3].

\footnotetext{
* Correspondence: jiao.yujuan@hotmail.com

${ }^{1}$ Department of Neurology, China-Japan Friendship Hospital, 2 Yinghua

Dongjie, Hepingli, 100029 Beijing, China

Full list of author information is available at the end of the article
}

Here, we report a Chinese HSP-TCC non-consanguineous family whose affected family members possess two compound heterozygous mutations in SPG11. These results implicate a need for wider screening of SPG11 mutations in ARHSP-TCC patients.

\section{Case presentation}

Index Patient (II:3) is a 26-year old female with healthy non-consanguineous parents (Fig. 1). Beginning at the age of 20 , she developed weakness in the lower limbs, accompanied by spasticity which was not alleviated by treatment with baclofen. She is cognitively impaired, advancing in her education only until middle school. MMSE and MoCA scores were $23 / 30$ and $15 / 30$, respectively (impaired in execution, calculation and delayed memory). Upon examination, she had mild dysarthria, bilateral patellar clonus, positive Babinski's sign, pes cavus (Fig. 2) and a scissors gait. Upper extremities were apparently normal. Blood and CSF testing showed no marked abnormalities. EMG revealed neurogenic, mild axonal sensory-motor neuropathy, 


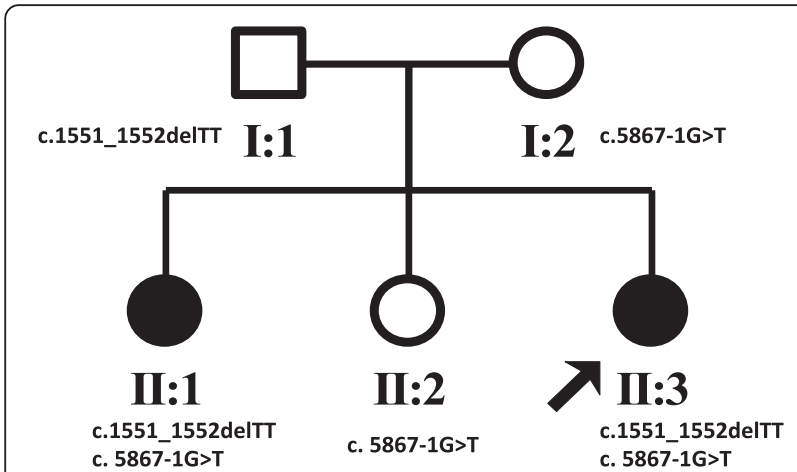

Fig. 1 Family pedigree of non-conanguineous parents indicating segregation of SPG11 mutations. Squares, male; circles, female; filled circle, affected female; arrow, proband

especially in lower extremities. Brain MRI revealed extreme thinning of corpus callosum, particularly in the anterior region (Fig. 3a). Thoracic MRI showed remarkable atrophy of thoracic spinal cord (Fig. 3b).

Her sister (II-1) is a 33-year old female with a similar, but more severe clinical phenotype with an 18 year history of disease. She is bedridden and severely handicapped due to the disturbance in her gait. She has severe

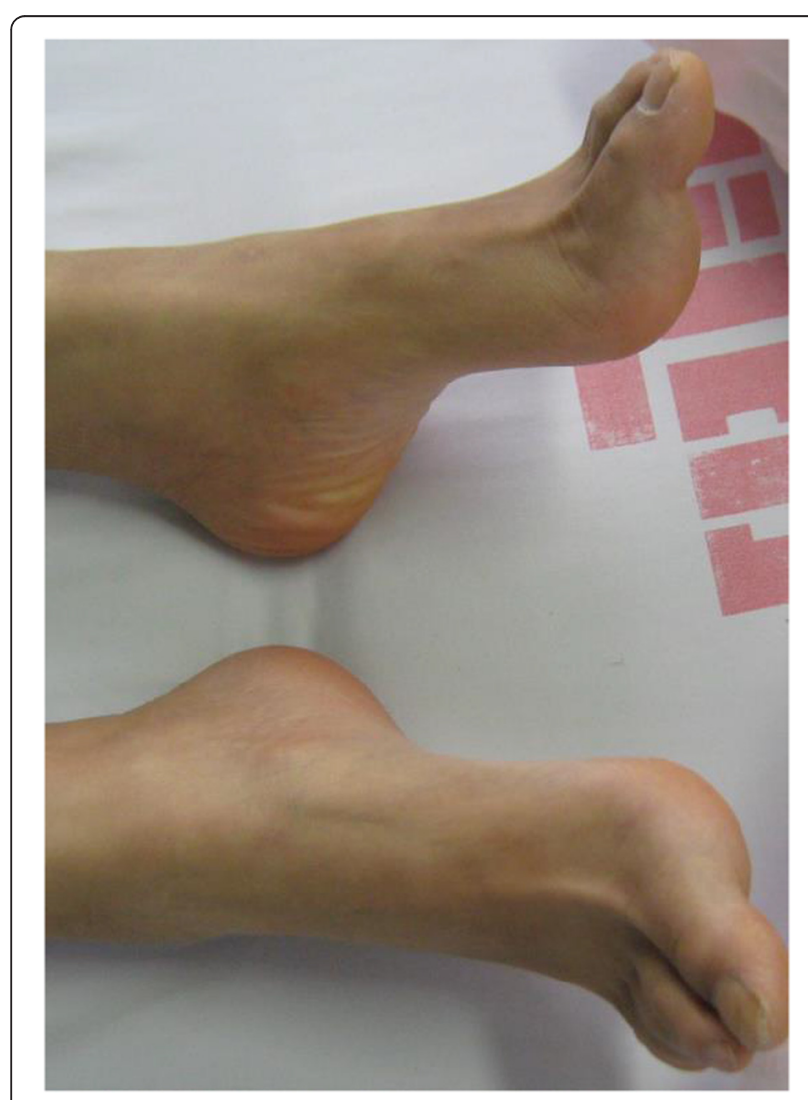

Fig. 2 Presentation of pes cavus in the index patient (II:3)

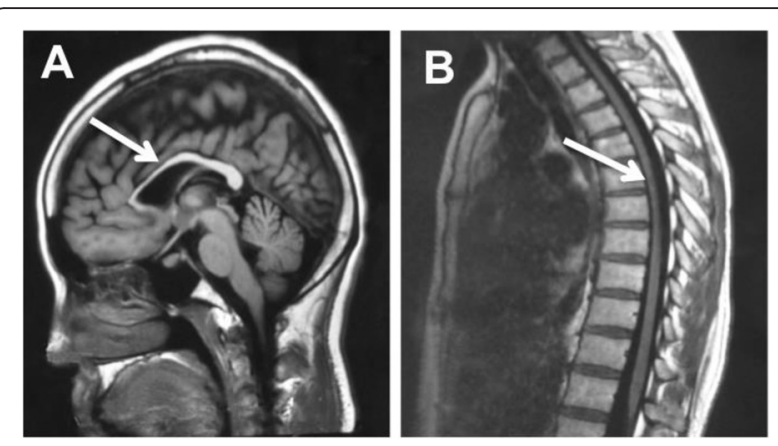

Fig. 3 Sagittal brain and thoracic spinal MRI image in index patient (II:3). a: Brain MRI shows thinning of corpus callosum, emphasized in the anterior part with a "beaked" shape (arrow). Ther is no obvious periventricular or deep cerebellar white matter lesions and no obvious cerebral or cerebellar atrophy. b: Throracic spinal MRI shows thinning of the thoracic spinal cord with volume loss but no obvious signal abnormalities in the cord

cognitive impairment and did not progress past an elementary school education.

Genomic DNA was extracted from peripheral blood samples according to standard procedures. SPG11 was screened for mutations by PCR amplification using previously described primer pairs [4] followed by Sanger sequencing of the amplicons (Additional file 1). In the two affected individuals, compound heterozygous mutations were identified in the SPG11 gene: c.1551_1552delTT, p.Cys518SerfsTer39 in exon 7; and c.5867-1G > T (IVS30$1 G>T$ ), p.Thr1956ArgfsTer15, a splice site mutation in intron 30 (showed in Fig. 4). Segregation of the genetic variants was validated in the other family members: the father (I:1) carried c.1551_1552delTT in the heterozygous state, while the mother (I:2) and the middle sister (II:2) carried c.5867-1G > T (IVS30-1G > T) in the heterozygous state (see Fig. 1). Both parents and the middle sister showed no symptom of spasticity or cognitive deterioration. The c.1551_1552delTT is novel but is similar to two previously described, nearby deletion mutations at c.1550_1551delTT (rs312262730) and c.1549_1550delCT (rs312262730), both of which are associated with SPG11 phenotypes. The second mutation, c.5867-1G $>\mathrm{T}$ (IVS30-1G > T), is also novel. Neither of these mutations was detected in 100 unrelated healthy Chinese control individuals or in the 1000 Genomes database.

Bioinformatic analysis of these mutations is predicted to prematurely truncate the protein product. The c.1551_ 1552delTT mutations causes a frameshift at amino acid reside 518 resulting in a premature stop codon (p.Cys51 8SerfsTer39). Such a mutation would result in a truncated protein but more likely would result in reduced transcript levels due to non-sense mediated decay. Similar mutations have been described in other cases of SPG11 [5]. The second mutation alters the consensus AG sequence at the 

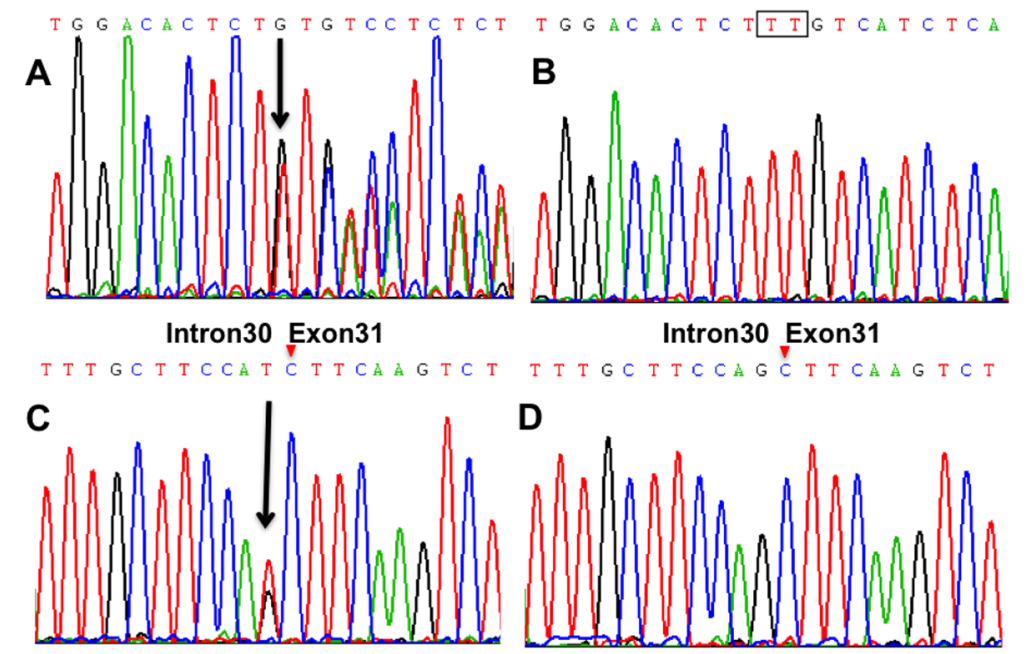

Fig. 4 Sanger sequencing of exon 7 (c.1551_1552delTT) containing a TT deletion in SPG11 in an affected individual (a) and unaffected individual (b). Sanger sequencing of a splice site mutation (c.5867-1G > T (IVS30-1G > T)) in intron 30 of SPG11 in an affected individual (c) and control, unaffected (d)

$3^{\prime}$ acceptor site of intron 30 (c.5867-1G > T (IVS30-1G > T)). Splice site predictions using the mutated sequence were performed using Splice Site Prediction by Neural Network (http://www.fruitfly.org/seq_tools/splice.html) and NetGene2 (http://www.cbs.dtu.dk/services/NetGene2/). Both analysis programs predict a complete abolishment of splicing using the mutated 3 ' acceptor site. Thus, we predict exclusion of exon 31 from the transcript resulting in a frameshift and premature stop codon shortly downstream (p.Tyr1956ArgfsTer15). Similar to the above mutation, this would likely result in a truncated protein or alternatively may result in reduced transcript levels due to nonsense mediated decay, suggesting a possible loss of function mechanism (Fig. 5).

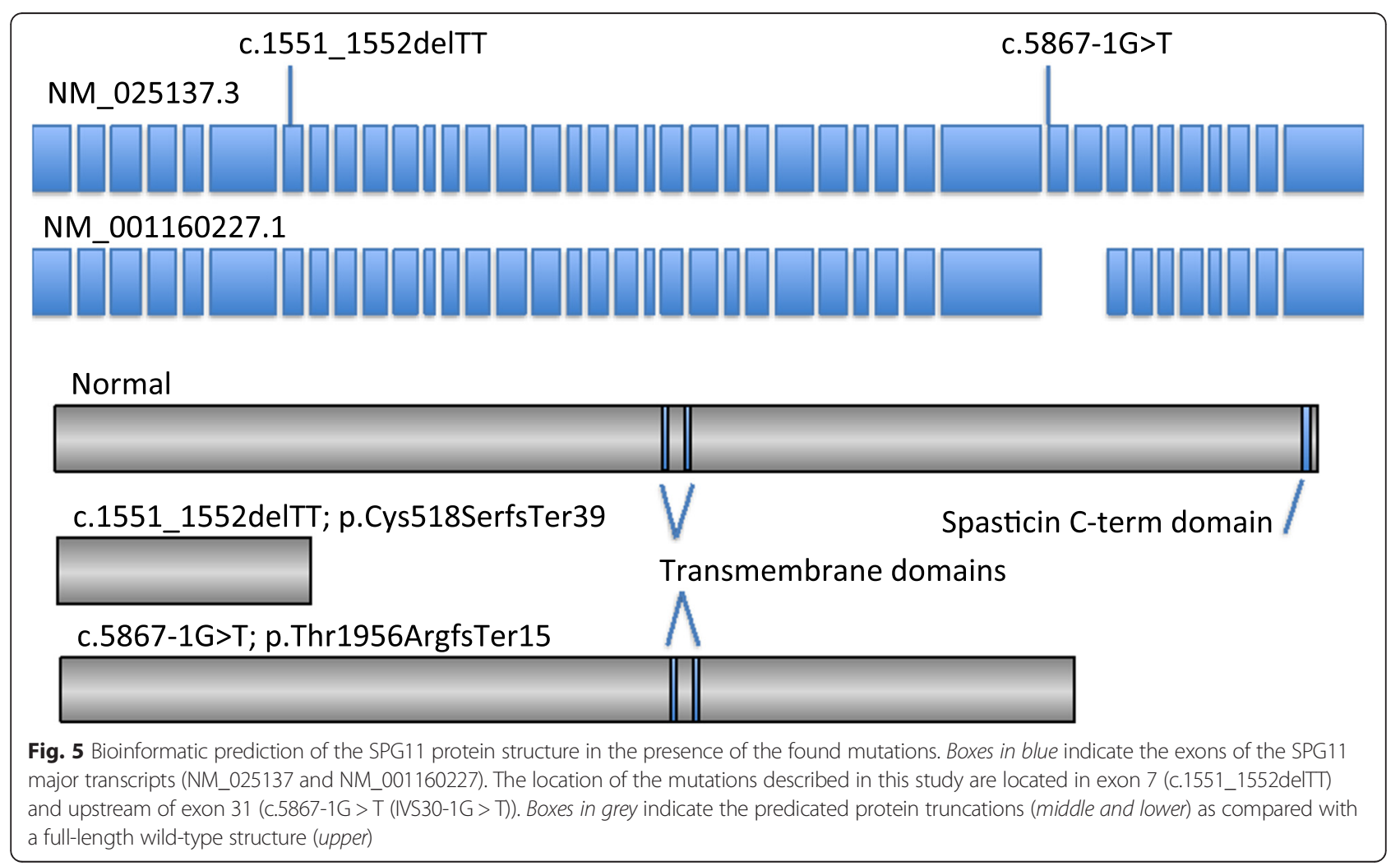




\section{Conclusions}

We identified novel compound heterozygous mutations in SPG11 in a Chinese HSP-TCC family (c.1551_1552delTT and c.5867-1G > T (IVS30-1G > T)). Our patients presented with typical clinical symptoms of ARHSP-TCC which included spastic paraplegia, cognitive impairment, peripheral neuropathy and distal musle atrophy. Thinning of thoracic spinal cord in our index patient is likely the result of degeneration of long corticospinal tracts and is usually present in nearly all subtypes of the HSP, mostly at later disease stages $[6,7]$.

Mutations in SPG11 are found in the majority of reported complex ARHSP-TCC cases with TCC being the single best indicator for SPG11 [2]. Another Chinese family with ARHSP-TCC was reported previously, the index patient presented with prominent intellectual disability rather than spasticity had different compound heterozygous mutations of SPG11 [8]. For patients presenting with HSP-TCC, SPG11 should be screened initially once infectious causes are eliminated. Other SPG loci including SPG15, SPG35 and SPG48 should be considered if no mutations in SPG11 are discovered [9]. Interestingly, mutations in SPG11 can also cause other disorders, such as juvenile amyotrophic lateral sclerosis (ALS5) [10], juvenile Parkinsonism [11], and autosomal recessive axonal Charcot-Marie-Tooth disease [12]. Thus, SPG11 mutations have an wide phenotypic spectrum suggesting that additional care should be taken when examining HSP patients. In summary, we demonstrate the importance of screening the SPG11 gene in the ARHSPTCC patients and have provided additional clinical phenotypes resulting from mutations in the SPG11 gene.

\section{Additional files}

Additional file 1: Primer sequences and reaction conditions used for the 40 exons of SPG11 gene. (DOCX $300 \mathrm{~kb}$ )

Additional file 2: Splice Site Prediction by Neural Network. (PPTX 77 kb)

\section{Abbreviations}

$A R$, autosomal recessive; CSF, cerebrospinal fluid; EMG, electromyography; HSP, hereditary spastic paraplegia; MMSE, mini-mental state examination; MoCA, Montreal cognitive assessment; MRI, magnetic resonance imaging; SPG, spastic paraplegia gene; TCC, thin corpus callosum

\section{Acknowledgements}

The authors would like to thank the patients and their families, without their participation this work would not have been possible. The authors would like to acknowledge Tetsuo Ashizawa for his advice for result analysis.

\section{Funding}

This study was supported in part by the Research Fund of the China-Japan Friendship Hospital (2013-RC-3).

\section{Authors' contributions}

Study design and drafting of manuscript: LZ, YJ. Clinical study perform: YJ, JJ. Genetic analysis: KNM LZ. Acquisition, and interpretation of data: YJ, KNM LZ, JJ. Critical revision of the manuscript: KNM YJ, LZ. Obtained funding: YJ. All authors have read and approved the final manuscript.

\section{Competing interests}

The authors declare that they have no competing interests.

Ethics approval and consent to participate

The study protocol was reviewed and approved by the Ethics Committee (IRB) of China-Japan Friendship Hospital (2016-7-1). Written informed consent was obtained from the patient and her family for genetic analysis and publication of this case report.

\section{Author details}

${ }^{1}$ Department of Neurology, China-Japan Friendship Hospital, 2 Yinghua Dongjie, Hepingli, 100029 Beijing, China. ${ }^{2}$ McKnight Brain Institute and the Department of Neurology, College of Medicine, University of Florida, Gainesville, FL 32610, United States of America.

Received: 5 December 2015 Accepted: 17 May 2016

Published online: 03 June 2016

\section{References}

1. Fink JK. Hereditary spastic paraplegia: clinico-pathologic features and emerging molecular mechanisms. Acta Neuropathol. 2013;126:307-28.

2. Stevanin G, Azzedine $H$, Denora $P$, Boukhris A, Tazie M, Lossos A, et al, Mutations in SPG11 are frequent in autosomal recessive spastic paraplegia with thin corpus callosum, cognitive decline and lower motor neuron degeneration. Brain. 2008;131:772-84.

3. Paisan-Ruiz C, Dogu O, Yilmaz A, Houlden H, Singleton A. SPG11 mutations are common in familial cases of complicated hereditary spastic paraplegia (HSP). Neurology. 2008;70:1384-9.

4. Stevanin G, Santorelli FM, Azzedine H, Coutinho P, Chomilier J, Denora PS, et al. Mutations in SPG11, encoding spatacsin, are a major cause of spastic paraplegia with thin corpus callosum. Nat Genet. 2007;39:366-72.

5. Samaranch L, Riverol M, Masdeu JC, Lorenzo E, Vidal-Taboada JM, Irigoyen J, et al. SPG11 compound mutations in spastic paraparesis with thin corpus callosum. Neurology. 2008;71:332-6.

6. Hourani R, El-Hajj T, Barada WH, Hourani M, Yamout BI. MR imaging findings in autosomal recessive hereditary spastic paraplegia. AJNR Am J Neuroradiol. 2009:30:936-40

7. Sperfeld AD, Baumgartner A, Kassubek J. Magnetic resonance investigation of the upper spinal cord in pure and complicated hereditary spastic paraparesis. Eur Neurol. 2005;54:181-5.

8. Ma J, Xiong L, Chang Y, Jing X, Huang W, Hu B, et al. Novel mutations C. [5121_5122insAG] + [6859C > T] of the SPG11 gene associated with cerebellum hypometabolism in a Chinese case of hereditary spastic paraplegia with thin corpus callosum. Parkinsonism Relat Disord. 2014;20(2):256-9.

9. Pensato V, Castellotti B, Gellera C, Pareyson D, Ciano C, Nanetti L, et al. Overlapping phenotypes in complex spastic paraplegias SPG11, SPG15, SPG35 and SPG48. Brain. 2014;137:1907-20.

10. Orlacchio A, Babalini C, Borreca A, Patrono C, Massa R, Basaran S, et al. SPATACSIN mutations cause autosomal recessive juvenile amyotrophic lateral sclerosis. Brain. 2010;133:591-8.

11. Anheim M, Lagier-Tourenne C, Stevanin G, Fleury M, Durr A, Namer IJ, et al. SPG11 spastic paraplegia. A new cause of juvenile parkinsonism. J Neurol. 2009;256:104-8

12. Montecchiani C, Pedace L, Lo Giudice T, Casella A, Mearini M, Gaudiello F, et al. ALS5/SPG11/KIAA1840 mutations cause autosomal recessive axonal Charcot-Marie-Tooth disease. Brain. 2016;139:73-85.

Submit your next manuscript to BioMed Central and we will help you at every step:

- We accept pre-submission inquiries

- Our selector tool helps you to find the most relevant journal

- We provide round the clock customer support

- Convenient online submission

- Thorough peer review

- Inclusion in PubMed and all major indexing services

- Maximum visibility for your research 